\title{
Effect of Ligninolytic Axenic and Coculture White- Rot Fungi on Rice Straw Chemical and In Vitro Fermentation Characteristics.
}

\author{
Osmond Datsomor \\ Yangzhou University \\ Zhao Gou-qi \\ Yangzhou University \\ Lin Miao ( $\square$ linmiao@yzu.edu.cn ) \\ Yangzhou University
}

\section{Research Article}

Keywords: Rice straw, White rot fungi, Coculture, solid state fermentation

Posted Date: August 3rd, 2021

DOl: https://doi.org/10.21203/rs.3.rs-763132/v1

License: (c) (i) This work is licensed under a Creative Commons Attribution 4.0 International License.

Read Full License 


\section{Abstract}

The objective of this study was to investigate the axenic culture of Pleurotus ostreatus, Phanerochaete chrysosporium, and the coculture ( $P$. chrysosporium and $P$. ostreatus) for their potential to break down lignin and to enhance the rumen fermentability of rice straw. Rice straw was fermented by two lignindegrading fungi, namely, $P$. ostreatus, $P$. chrysosporium, and coculture ( $P$. ostreatus and $P$. chrysosporium) under solid-state fermentation (SSF). The coculture exhibited a mutual intermingling plus inhibition interaction. Coculture treated straw had a lower lignin content (5.26\%) compared to $P$. chrysosporium axenic treated straw $(6.18 \%)$, although $P$. ostreatus axenic treated straw was the lowest $(3.27 \%)$. The polysaccharide content of coculture treated straw was higher than $P$. chrysosporium axenic treated straw, although smaller than $P$. ostreatus. $P$. ostreatus and coculture suitably delignified rice straw without adversely affecting cellulose. Treatment of rice straw with coculture improved in vitro dry matter digestibility (68.08\%), total volatile fatty acids (35.27\%), and total gas $(57.4 \mathrm{ml} / 200 \mathrm{mg})$ compared to $P$. chrysosporium $(45.09 \%, 32.24 \%, 44.39 \mathrm{ml} / 200 \mathrm{mg}$ ) but was second to $P$. ostreatus $(75.34 \%, 38.31 \%$, $65.60 \mathrm{ml} / 200 \mathrm{mg}$ ). The coculture via synergistic interaction has the potential to decompose lignin and improve the nutritive value of rice straw than $P$. chrysosporium.

\section{Introduction}

Rice straw is an abundant and inexpensive source of energy-rich roughage residue. Therefore, it is used as a part of the ration of ruminant animals (cattle, buffalo, goat, and sheep) in most rice-cultivating countries ${ }^{1}$, especially in the dry season when fresh forage is limited. Rice straw contains $25-45 \%$ cellulose, $25-30 \%$ hemicellulose, and 10-15\% lignin. However, the proportion utilized is limited owing to its high lignin and low protein content ${ }^{2}$. Lignin encapsulates structural polysaccharides preventing its hydrolysis within the rumen. To increase rice straw utilization in the diet of ruminant, lignin and its limitations have to be addressed. Delignification, which encompasses complete, partial removal, and modification of lignin, is central to rice straw pretreatment.

Physical, chemical, and/ biological delignification treatments have been established to increase the nutritive value, nutrient digestibility, and utilization of rice straw. However, biological pretreatment is the most preferable because it is practical, safe, and environmentally friendly ${ }^{3}$. Research is mainly focused on using white-rot fungi as they are the only organism known to effectively and efficiently mineralize lignin into water and carbon dioxide ${ }^{4}$. Several white-rot fungi, including $P$. ostreatus and $P$. chrysosporium, have been screened under axenic culture condition to exhibit high ligninolytic potential ${ }^{5}$. However, in nature, fungi typically live and grow near each other and establish multiple spectrums of interactions among themselves, with abiotic factors in the environment and substrate. Species can form synergistic interactions to coordinate substrate structural polysaccharide and lignin degradation via powerful enzyme cascade ${ }^{6}$.

P. ostreatus and P. chrysosporium are white-rot fungi with high ligninolytic capability. Chen, et al. ${ }^{7}$ reported higher degradation ratio of lignin (26.38\%) and cellulose (33.29\%) using a P. chrysosporium and 
T. viride coculture. Tobacco stalk substrate treated with $T$. hirsuta $\mathrm{S} 13$ and $P$. ostreatus $\mathrm{S} 18$ coculture also achieved a 2-fold lignin degradation rate (from 23.7 to $41.1 \%)^{8}$. Recently, Kaur, et al. ${ }^{9}$ reported a maximum laccase ( $\mathrm{LaC})$, lignin peroxidase ( $\mathrm{LiP})$, and manganese peroxidase (MnP) activities of 2.40 nkat/gds, 37.92 nkat/gds, and 62.50 nkat/gds respectively via a Pleurotus ostreatus and Phanerochaete chrysosporium fungal consortium on rice straw. These independent studies demonstrate the potential of white-rot fungal consortium to alter cell wall chemical constituents of various straw via their enzyme activities/secretions.

These independent studies, however did not ascertain the type of antagonist interaction and in vitro feed evaluation assay including digestibility, volatile fatty acids and in vitro gas production of coculture and axenic white-rot fungi species which is a critical step to in vivo and feeding evaluation. Therefore, in this study axenic cultures of white-rot fungi $P$. ostreatus, $P$. chrysosporium, and coculture were employed to delignified rice straw, classify the nature of the interaction, effect of both axenic and coculture on chemical fiber component degradation, in vitro dry matter digestibility, volatile fatty acids, and in vitro gas volume production. This would provide useful information on ration formulation strategies using whiterot treated rice straw for ruminant nutrition during the dry season.

\section{Materials And Methods}

\section{Fungi}

P. chrysosporium CGMCC 3.7212 and P. ostreatus CGMCC 3.7292 axenic white-rot fungi were used for the study. They were provided by China Agricultural University in Beijing, China, grown in a malt extract agar (MEA) plate culture-medium (malt extract $20 \mathrm{~g}$; agar $20 \mathrm{~g}$; nutritional yeast $2 \mathrm{~g}$; per $\mathrm{L}$ ) and stored at 4 ${ }^{\circ} \mathrm{C}$. P. chrysosporium and $P$. ostreatus are ligninolytic white-rot fungi thus have the ability to degrade lignin. Agar plates were prepared using an autoclaved sterilized MEA (malt extract $20 \mathrm{~g}$; agar $20 \mathrm{~g}$; nutritional yeast $2 \mathrm{~g}$; per $\mathrm{L} ; 121^{\circ} \mathrm{C}$ to $25 \mathrm{~min}$ ) and inoculated with a $0.5 \mathrm{~cm}^{2}$ piece of the fungi at $25 \pm 1{ }^{\circ} \mathrm{C}$ incubation until mycelium covered the entire MEA plates surface.

\section{Paired interaction on agar plates}

Interspecies interactions of the two fungal species were investigated by placing square inoculum plugs of the same size at two opposite pole of MEA plates $40 \mathrm{~mm}$ apart. Interactions between opposing mycelia were assessed visually every second day using the protocol of Rayner and Boddy ${ }^{10}$.

\section{Millet spawn preparation}

Millet grains were washed in water and boiled for $15 \mathrm{~min}$. The boiled grains were transferred onto a sieve to drain. The grains were packed into two polyethylene mycobag ( $25 \mathrm{~cm}$ wide and $37 \mathrm{~cm}$ height) until it was three quarters full and then autoclaved at $121^{\circ} \mathrm{C}$ for $45 \mathrm{~min}$. The content of each mycobag was permitted to cool and then separately inoculated aseptically with five $1 \mathrm{~cm}^{2}$ pieces of mycelium agar and sealed. The polyethylene mycobags content was shaken by hand for uniform mixing of the mycelium 
with grains and incubated in a ventilated incubator at $25 \pm 1^{\circ} \mathrm{C}$ until the mycelia colonized all grains. The spawns were stored in a cold room at $4^{\circ} \mathrm{C}$ to stop the mycelia from further growth and for future use.

\section{Substrate preparation and inoculation}

The rice straw used in the present study was collected from the rice field of Yangzhou University, Jiangsu, China. Rice straw was chopped into 2 to $3 \mathrm{~cm}$ lengths and packed into a $2 \mathrm{~mm}$ net mesh. The packed rice straw was placed into a barrel with water of about three times the weight of the straw and left overnight for the water to penetrate the inner structures of the straw. The soaked rice straw was hanged in the open air via a hanger to allow excess water to drain from the straw. $250 \mathrm{~g}$ of the wet substrate was weighed into 16 polyethylene mycobags and sterilized in an autoclave at a temperature of $121^{\circ} \mathrm{C}$ for $1 \mathrm{~h}$. The autoclaved mycobags were cooled to room temperature and each straw mycobag inoculated with millet spawn at $5 \%(\mathrm{w} / \mathrm{w})$ of straw $(10 \mathrm{~g})$.

The inoculated mycobags were shaken to ensure uniform distribution of spawn and incubated at a temperature of $25 \pm 1{ }^{\circ} \mathrm{C}$ and $75-80 \%$ humidity. The mycobags were incubated for 30 days. The control group consisted of uninoculated rice straw (CT), and the experimental group being rice straw treated with axenic culture of Pleurotus ostreatus (PO), Phanerochaete chrysosporium (PC) white-rot fungi, and coculture (CC). All of the experiments were performed in quadruplicate. After incubation, the mycobags with treated substrate including mycelium was oven-dried at $64^{\circ} \mathrm{C}$ for $48 \mathrm{~h}$. The dried fungi treated rice straw were ground over a $1 \mathrm{~mm}$ sieve using a CM100 miller to obtain a homogenous sample and stored for further chemical and in vitro analysis.

\section{Chemical fiber analysis}

The samples were analyzed for dry matter by drying at $105^{\circ} \mathrm{C}$ in an oven dryer for $3 \mathrm{~h}$. The neutral detergent fiber (NDF), acid detergent fiber (ADF), cellulose, hemicellulose and acid detergent lignin (ADL) analysis was performed as described by Van Soest, et al. ${ }^{11}$ employing an Ankom 2000 automated fiber analyzer (ANKOM Technology, Mecedon, New York, USA). Samples $(0.5-1 \mathrm{~g})$ were placed into polyester mesh bags (ankom F57) and sealed. Bags and $2000 \mathrm{ml}$ of neutral detergent were put into the automatic fiber analyzer at $100{ }^{\circ} \mathrm{C}$ for $60 \mathrm{~min}$. Then, the bags were washed to neutral with distilled water, dried and weighed. Dried residue was represented as NDF. Remaining samples and $2000 \mathrm{ml}$ of acid detergent were put into the automatic fiber analyzer at $100{ }^{\circ} \mathrm{C}$ for $60 \mathrm{~min}$. Then, the bags were washed to neutral with distilled water, dried and weighed. Dried residue was represented as ADF. The content of hemicellulose was calculated as the difference between NDF and ADF. Dried residue was soaked in $72 \%(\mathrm{v} / \mathrm{v}) \mathrm{H}_{2} \mathrm{SO}_{4}$ and kept at $25^{\circ} \mathrm{C}$ for $2 \mathrm{~h}$. Thereafter, the bags were washed to neutral with distilled water, dried and weighed. The remaining samples was kept at $550^{\circ} \mathrm{C}$ for $3 \mathrm{~h}$ in a tared crucible and reweighed to calculate the loss as ADL. The content of cellulose was calculated as the difference between ADF and ADL. Ash content was determined by carbonization of the samples in a muffle furnace at $550{ }^{\circ} \mathrm{C}$ for at least $3 \mathrm{~h}$. Organic matter (OM) was calculated as the difference between DM and ash content. All calculations were on a dry matter basis expressed in percentages. 


\section{In vitro fermentation}

The effect of fungal treatment was evaluated using in vitro ruminal fermentation, according to the procedure of Menke, et al. ${ }^{12}$.

Briefly, fresh rumen fluid collected from three rumen-fistulated Holstein cow fed corn silage and oat strawbased diet was mixed with a buffer solution (Buffer A: $13.2 \mathrm{~g} \mathrm{CaCl} \cdot 2 \mathrm{H}_{2} \mathrm{O}, 10.0 \mathrm{~g} \mathrm{MnCl}_{2} \cdot 4 \mathrm{H}_{2} \mathrm{O}, 1.0 \mathrm{~g}$ $\mathrm{CoCl}_{2} \cdot 6 \mathrm{H}_{2} \mathrm{O}$ and $8.0 \mathrm{~g} \mathrm{FeCl}_{3} \cdot 6 \mathrm{H}_{2} \mathrm{O}$ per $100 \mathrm{ml}$; Buffer B: $35.0 \mathrm{~g} \mathrm{NaHCO}_{3}$ and $4.0 \mathrm{~g} \mathrm{NH}_{4} \mathrm{HCO}_{3}$ per $1000 \mathrm{ml}$;

Buffer C: $5.7 \mathrm{~g} \mathrm{Na}_{2} \mathrm{HPO}_{4}$ and $0.6 \mathrm{~g} \mathrm{MgSO}_{4} \cdot 7 \mathrm{H}_{2} \mathrm{O}$ and $6.2 \mathrm{~g} \mathrm{KH}_{2} \mathrm{PO}_{4}$ per $1000 \mathrm{ml}$ ) in a 1:2 (v/v) ratio under continuous flushing with $\mathrm{CO}_{2} .200 \mathrm{mg}$ of an oven-dried control group and experimental group samples were weighed into a $100 \mathrm{ml}$ glass vial. Each glass vial received $30 \mathrm{ml}$ of buffered rumen fluid and was incubated in a thermo-static shaker at $39^{\circ} \mathrm{C}$ for $48 \mathrm{~h}$ along with blanks. All the groups (control, experimental, and blank) were conducted in quadruplicate.

\section{In vitro gas, volatile fatty acids and dry matter digestibility}

In vitro gas produced was recorded using a digital pressure gauge at $0,3,6,12,24,36$ and $48 \mathrm{~h}$ of incubation. The kinetics of in vitro gas production was based on net gas volume (gas of sample - the gas of blank). The vials were taken out of the incubator at $48 \mathrm{~h}$ and placed into an ice-water bath to stop fermentation. The fermentation mixture $\mathrm{pH}$ was measured using a $\mathrm{PHS}-3 \mathrm{C} \mathrm{pH}$ meter and centrifuged using Eppendorf centrifuge 5810R at $8000 \times \mathrm{g}$ and $4{ }^{\circ} \mathrm{C}$ for $15 \mathrm{~min}$ to obtain a supernatant and nonfermented solid residue. Each sample's supernatant was transferred into centrifuge tubes (1.5 ml) and used for volatile fatty acids (VFA) concentration determination. The VFA profile was determined using a GC 3420 gas chromatography. The non-fermentable solid residues of each sample were dried at $65^{\circ} \mathrm{C}$ overnight and weighed. The in vitro dry matter digestibility (IVDMD) was estimated as the weight difference between the dried non-fermentable solid residues and initial weight.

\section{Statistical analysis}

The effects of axenic culture white-rot fungal species and coculture on the chemical composition, $\mathrm{pH}$, VFA profile, in vitro gas of control, and experimental group samples, were analyzed as a single factor ANOVA using SPSS, version 21.0 (IBM Corp., Armonk, NY, USA). Post-hoc multiple comparisons with Duncan's significant test at a significance level of 0.05 was performed to determine the significance between experimental groups. Prior to conduction the ANOVA, the assumption of homogeneity of variances was tested and satisfied based on Levene's test $(P>0.05)$.

\section{Results}

\section{Interactions between P. ostreatus and P. chrysosporium fungal isolates in dual culture}

To determine the type of interaction that occurs between $P$. ostreatus and $P$. chrysosporium, a visual mycelium confrontation test was carried out. After plating (Fig. 1A), $P$. chrysosporium was observed to 
grow faster than $P$. ostreatus (Fig. 1B). P. chrysosporium and P. ostreatus mycelia made initial contact leading to the formation of a distinct dense whitish barrage (Fig. 1C). The whitish barrage formed is evident of a mutual intermingling owing to cytoplasmic contact of the fungi isolates via hyphal fusion and subsequent increased continual fusion of mycelia mass (anastomosis). A brownish to yellow coloration of the barrage was observed and growth of barrage mycelia towards $P$. ostreatus and $P$. chrysosporium was restricted resulting in the formation of a partial inhibition region (Fig. 1D). The axenic white-rot fungus species and coculture all thrived well on rice straw with no mold visibly detected (Fig. 2A, $B, C)$.

\section{Chemical fiber composition of rice straw treated with P. ostreatus axenic fungi, P. chrysesporium axenic culture and coculture}

Treatments of rice straw with $P$. ostreatus, $P$. chrysosporium and coculture significantly $(P<0.05)$ affected rice straw chemical composition and cell wall content (Table 1). The dry matter value of $P$. ostreatus treated rice straw was significantly $(P<0.001)$ higher than $P$. chrysosporium and coculture treated rice straw but significantly $(P<0.001)$ less than control. Coculture treated rice straw had a significantly $(P<0.001)$ higher DM value than $P$. chrysosporium treated rice straw.

Table 1

Chemical composition (\%) of rice straw after incubation with axenic and coculture white-rot fungi.

\begin{tabular}{|lllllll|}
\hline Parameters & Control & $P$. ostreatus & $P$. chrysosporium & Coculture & SEM & $P$ value \\
\hline DM & $94.25^{\mathrm{a}}$ & $88.48^{\mathrm{b}}$ & $81.58^{\mathrm{d}}$ & $84.73^{\mathrm{c}}$ & 1.23 & $<0.001$ \\
\hline OM & $81.23^{\mathrm{a}}$ & $73.2^{\mathrm{b}}$ & $62.8^{\mathrm{d}}$ & $68.81 \mathrm{c}$ & 1.74 & $<0.001$ \\
\hline Ash & $13.02^{\mathrm{d}}$ & $15.2^{\mathrm{c}}$ & $18.7^{\mathrm{a}}$ & $15.93^{\mathrm{b}}$ & 0.53 & $<0.001$ \\
\hline NDF & $70.63^{\mathrm{a}}$ & $61.00^{\mathrm{b}}$ & $54.44^{\mathrm{d}}$ & $57.12^{\mathrm{c}}$ & 1.58 & $<0.001$ \\
\hline ADF & $51.82^{\mathrm{a}}$ & $45.46^{\mathrm{b}}$ & $39.39^{\mathrm{d}}$ & $42.27^{\mathrm{c}}$ & 1.19 & $<0.001$ \\
\hline ADL & $8.16^{\mathrm{a}}$ & $3.27^{\mathrm{d}}$ & $6.18^{\mathrm{b}}$ & $5.26^{\mathrm{c}}$ & 0.45 & $<0.001$ \\
\hline Cellulose & $43.66^{\mathrm{a}}$ & $42.1^{\mathrm{b}}$ & $33.21^{\mathrm{d}}$ & $37.00^{\mathrm{c}}$ & 1.08 & $<0.001$ \\
\hline Hemicellulose & $18.81^{\mathrm{a}}$ & $15.54^{\mathrm{b}}$ & $15.08^{\mathrm{b}}$ & $14.90^{\mathrm{b}}$ & 0.42 & $<0.001$ \\
\hline $\begin{array}{l}\text { SEM, standard error of the mean. } \\
\text { significant difference }(P<0.05) . \text { Each values with different superscript letters in a row represent }\end{array}$ \\
\hline
\end{tabular}

Among all the treatment groups, $P$. chrysosporium treated straw had significantly $(P<0.001)$ the lowest DM value. 
A similar trend was observed in the OM, NDF, ADF, and cellulose content. Ash value of $P$. ostreatus treated rice straw was significantly $(P<0.001)$ lower than $P$. chrysosporium and coculture treated rice straw but significantly $(P<0.001)$ higher than control. Coculture treated rice straw ash value was significantly $(P<$ $0.001)$ less than $P$. chrysosporium treated rice straw. Compared to $P$. ostreatus, coculture, and control ash value, $P$. chrysosporium treated rice straw was significantly $(P<0.001)$ the greatest. The ADL value of $P$. ostreatus treated rice straw was significantly $(P<0.001)$ the least compared to control, $P$. chrysosporium, and coculture treated rice straw. Coculture treated rice straw ADL value was significantly $(P<0.001)$ lower than $P$. chrysosporium treated rice straw and control. The hemicellulose value of $P$. ostreatus, $P$. chrysosporium, and coculture treated rice straw were significantly $(P<0.001)$ lower than control.

\section{pH, IVDMD and volatile fatty acids profile of rice straw treated with P. ostreatus axenic fungi, $\mathrm{P}$. chrysosporium axenic culture and coculture}

Rice straw treated with $P$. ostreatus, $P$. chrysosporium and coculture did not have a significant $(P>0.41)$ effect on pH but IVDMD, total VFA, and individual VFA were significant $(P<0.05)$ (Table 2$)$. Compared to control, $P$. chrysosporium and coculture, $P$. ostreatus treated rice straw IVDMD was significantly $(P<$ $0.001)$ the highest. IVDMD of coculture treated rice straw was significantly $(P<0.001)$ higher than control and $P$. chrysosporium. $P$. chrysosporium treated rice straw recorded significantly $(P<0.001)$ the least IVDMD compared to control, $P$. ostreatus, $P$. chrysosporium, and coculture. A similar observation was made in total VFA. $P$. ostreatus treated rice straw acetate value was significantly $(P<0.001)$ lower than control but significantly $(P<0.001)$ higher than $P$. chrysosporium and coculture treated rice straw. The acetate value of coculture treated rice straw was significantly $(P<0.001)$ higher than $P$. chrysosporium but significantly $(P<0.001)$ lower than control. Compared to control, $P$. ostreatus and coculture, $P$. chrysosporium acetate value were significantly $(P<0.001)$ the least. A similar trend was observed in butyrate content. Propionate content of $P$. ostreatus treated rice straw was significantly $(P<0.001)$ higher than control, $P$. chrysosporium and coculture treated rice straw. Coculture treated rice straw propionate was significantly $(P<0.001)$ higher than $P$. chrysosporium and control. Among all the treatment groups, propionate content of $P$. chrysosporium was significantly $(P<0.001)$ the least. 
Table 2

$\mathrm{pH}$, IVDMD and VFA from rice straw after incubation with axenic and coculture white-rot fungi.

\begin{tabular}{|c|c|c|c|c|c|c|}
\hline Parameter & Control & P. ostreatus & P. chrysosporium & Coculture & SEM & $P$ value \\
\hline $\mathrm{pH}$ & 6.84 & 6.85 & 6.97 & 6.69 & 0.03 & 0.41 \\
\hline IVDMD (\%) & $51.02^{c}$ & $75.34^{a}$ & $45.09^{d}$ & $68.08^{b}$ & 3.18 & $<0.001$ \\
\hline Total VFA (mM) & $34.38^{c}$ & $38.31^{a}$ & $32.24^{d}$ & $35.27^{b}$ & 0.56 & $<0.001$ \\
\hline \multicolumn{7}{|l|}{$\begin{array}{l}\text { Individual VFA } \\
\text { (\%Total VFA) }\end{array}$} \\
\hline Acetate & $67.06^{\mathrm{a}}$ & $66.13^{b}$ & $64.21^{d}$ & $65.03^{c}$ & 0.28 & $<0.001$ \\
\hline Propionate & $22.04^{c}$ & $25.16^{a}$ & $20.19^{d}$ & $23.04^{b}$ & 0.46 & $<0.001$ \\
\hline$A: P$ & $3.09^{a}$ & $2.63^{d}$ & $3.18^{b}$ & $2.83^{c}$ & 0.05 & $<0.001$ \\
\hline Isobutyrate & $1.08^{\mathrm{d}}$ & $2.50^{c}$ & $4.88^{\mathrm{a}}$ & $3.09^{b}$ & 0.35 & $<0.001$ \\
\hline Butyrate & $6.02^{a}$ & $5.34^{b}$ & $4.21^{d}$ & $5.09^{c}$ & 0.17 & $<0.001$ \\
\hline Isovalerate & $3.09^{c}$ & $1.09^{d}$ & $4.21^{\mathrm{a}}$ & $3.14^{b}$ & 0.29 & $<0.001$ \\
\hline Valerate & $1.13^{b}$ & $0.52^{b}$ & $2.43^{a}$ & $1.05^{b}$ & 0.23 & $<0.001$ \\
\hline
\end{tabular}

A:P ratio of $P$. ostreatus treated rice straw was significantly $(P<0.001)$ the lowest compared to control, $P$. chrysosporium, and coculture treated rice straw. Coculture treated rice straw A:P was significantly $(P<$ $0.001)$ lower than control and $P$. chrysosporium treated rice straw. $P$. chrysosporium treated rice straw A:P ratio was significantly $(P<0.001)$ the highest among all groups. Isobutyrate content of coculture treated rice straw was significantly $(P<0.001)$ higher than control, and $P$. ostreatus treated rice straw but significantly $(P<0.001)$ lower than $P$. chrysosporium treated rice straw. $P$. ostreatus isobutyrate content was significantly $(P<0.001)$ higher than control but significantly $(P<0.001)$ lower than $P$. chrysosporium and coculture treated rice straw. Among all the other treatments, $P$. chrysosporium had a significantly $(P$ $<0.001)$ higher isobutyrate content. Coculture treated rice straw isovalerate content was significantly $(P<$ $0.001)$ higher than $P$. ostreatus and control but significantly $(P<0.001)$ lower than $P$. chrysosporium treated rice straw. $P$. ostreatus and $P$. chrysosporium isovalerate content was significantly $(P<0.001)$ the least and highest among all the other treatment groups. $P$. chrysosporium treated rice straw had significantly $(P<0.001)$ the highest valerate content compared to control, $P$. ostreatus, and coculture treated rice straw. 
In vitro gas volume of rice straw treated with P. ostreatus axenic fungi, P. chrysosporium axenic culture and coculture

Significant difference $(P<0.001)$ existed between fungi treated rice straw groups at each incubation time interval (Table 3 ). The gas volume of $P$. ostreatus and $P$. chrysosporium treated rice straw was significantly $(P<0.001)$ the highest and the lowest. The gas volume of coculture treated rice straw was significantly $(P<0.001)$ higher than $P$. chrysosporium and control.

Table 3

In vitro gas volume from rice straw after incubation with axenic and coculture white-rot fungi.

\begin{tabular}{|lllllll|}
\hline $\begin{array}{l}\text { Gas volume } \\
\text { (ml/200 mg) }\end{array}$ & Control & $\boldsymbol{P}$. ostreatus & P. chrysosporium & Coculture & SEM & $P$ value \\
\hline Gv 3 h & $3.33^{\mathrm{c}}$ & $4.62^{\mathrm{a}}$ & $2.34^{\mathrm{d}}$ & $4.27^{\mathrm{b}}$ & 0.24 & $<0.001$ \\
\hline Gv 6 h & $13.07^{\mathrm{c}}$ & $15.61^{\mathrm{a}}$ & $10.55^{\mathrm{d}}$ & $13.42^{\mathrm{b}}$ & 0.49 & $<0.001$ \\
\hline Gv $12 \mathrm{~h}$ & $23.32^{\mathrm{c}}$ & $28.07^{\mathrm{a}}$ & $20.11^{\mathrm{d}}$ & $25.32^{\mathrm{b}}$ & 0.75 & $<0.001$ \\
\hline Gv 36 h & $49.06^{\mathrm{c}}$ & $63.25^{\mathrm{a}}$ & $43.08^{\mathrm{d}}$ & $54.80^{\mathrm{b}}$ & 1.92 & $<0.001$ \\
\hline Gv $48 \mathrm{~h}$ & $51.41^{\mathrm{c}}$ & $65.60^{\mathrm{a}}$ & $44.39^{\mathrm{d}}$ & $57.40^{\mathrm{b}}$ & 2.02 & $<0.001$ \\
\hline $\begin{array}{l}\text { SEM, standard error of the mean; Gv, Gas volume. } \\
\text { represent significant difference }(P<0.05) . \text { Each value was the mean of four replicates. }\end{array}$ & \\
\hline
\end{tabular}

\section{Discussion}

The species interaction observation is consistent with the report that instances where mutual intermingling coexist with inhibition, a barrage is formed ${ }^{13}$. The coloration of interacting mycelia fronts arises from melanin biosynthesis and improved phenoloxidase activity in the interacting hyphal tip region ${ }^{14}$. Coloration may be a signal of free radicals present in the hyphal tip region which invigorate lignin and lignin-type polymer decomposition ${ }^{15}$. The vigorous growth of fungi on the substrate is a precondition for the fermentation process to occur. The rapid growth of mycelia permits inoculated fungi to predominant the substrate, inhibiting unwanted microorganisms (molds or bacteria) from contaminating the substrate ${ }^{16}$.

There was a general decrease in DM for all the fungi treatments at the end of the experiment. The loss/ decline is unavoidable because fungi consume nutrient (fiber, protein, minerals, carbohydrates, and other nutrients) present within substrate dry matter for their self-proliferation and growth. In the present study, among the axenic fungi treated rice straw, $P$. chrysosporium and $P$. ostreatus had the most negligible and 
most DM content, which is similar to the report of Zheng, et al. ${ }^{17}$. This is due to the relatively fast growth of $P$. chrysosporium compared to the much slower growth exhibited by $P$. ostreatus ${ }^{18}$.

P. chrysosporium and P. ostreatus treated rice straw resulted in an organic matter loss of $23 \%$ and $10 \%$, respectively. This trend is similar to the report by Kerem, et al. ${ }^{18}$ who after 28 days treatment of cotton stalk with $P$. chrysosporium and $P$. ostreatus observed a $55 \%$ loss and $20 \%$ loss of organic matter although losses were much higher than the present study. Losses in organic matter by fungi during solid state fermentation (SSF) were primarily due to macronutrient molecules consumption (carbohydrate, fiber, protein, fat/lipids), primarily associated with the cell wall. The organic matter loss of the coculture was $15 \%$. Ash constitutes the inorganic matter that is additionally tricky for animal digestion however necessary in minute quantity. Consumption of organic matter by fungi leads to increased ash concentrations of the remaining dry matter. The ash content of fungal treated rice straw increased compared to the control indicating that the fungi did not utilize the residual ash constituents; thus, the fungus causing minimum $\mathrm{OM}$ also contributes to lower ash content and vice versa. A higher ash content was evident in rice straw degraded by $P$. chrysosporium, while $P$. ostreatus had a lower ash content. This observation agrees with the high ash residue in $P$. chrysosporium biodegraded wheat straw than $P$. ostreatus $^{19}$.

The axenic culture and coculture fungi substantially degraded fibrous fraction (NDF, ADF and ADL) content of the rice straw after 30 days of treatment which indicates that white-rot fungi as biological pretreatment are specialists in degrading structural carbohydrate and lignin in lignocellulosic tissues deriving organic carbon for their energy demands. In the current study, P. chrysosporium treated rice straw had lower cellulose but higher lignin content. This agrees with the considerable higher lignin and lower cellulose in biodegraded wheat straw ${ }^{20}$. According to Salvachúa, et al. ${ }^{21}$ proteomic assay of the secretome of $P$. chrysosporium revealed several diverse glycoside hydrolases (GHs) made up of a cascade of enzymes involved in the absolute breakdown of cellulose ${ }^{21}$. P. chrysosporium is therefore termed a non-selective/simultaneous delignifier ${ }^{22}$ thus consumes a large amount of cellulose with a small loss in lignin or shows no preference to lignocellulosic. Moreover, the low cellulose but high lignin content in $P$. chrysosporium treated rice straw illustrate that excessive degradation of cellulose decelerates lignin degradation.

In contrast, $P$. ostreatus demonstrated a considerably higher cellulose content and lower lignin which is similar to the report of $\mathrm{Nie}$, et al. ${ }^{23}$. This might be because $P$. ostreatus produces diverse ligninolytic enzymes that exclusively attack lignin over cellulose ${ }^{24}$. P. ostreatus is termed a selective white-rot fungus as decomposing of lignin is associated with a marginal loss in cellulose ${ }^{25}$.

Species can form synergistic interaction to coordinate substrate degradation ${ }^{14}$. Lignin and cellulose and other cell wall constituent (DM, OM, NDF, ADF) of coculture treated rice straw were intermediate of $P$. ostreatus and $P$. chrysosporium, thus superior to $P$. chrysosporium but inferior to $P$. ostreatus, which is an indication of synergistic effect. Coculture compared to single microorganisms attain superior growth, 
efficient biological processes and enzymatic activities via synergistic mechanism which accelerates the delignification process ${ }^{26}$. A coculture of $P$. ostreatus and $P$. chrysosporium, did not show synergistic effect in the degradation of lignocelluloses ${ }^{6}$ which is contrary to the current study. This could be due to differences in substrate types (coffee) and fermentation medium (liquid) utilized.

The $\mathrm{pH}$ is a vital index reflecting rumen environment. In the present study, axenic and coculture treatment of rice straw did not alter rumen $\mathrm{pH}$ which is similar with the finding of Khonkhaeng and Cherdthong ${ }^{27}$. The values in the current study were all within the usual range $(>6.3)$ for optimal rumen metabolism ${ }^{28}$. The degree of lignification or higher lignin content directly affects the reduction of rice straw's nutrient digestibility. Removal of lignin is directly associated with enzymatic digestion and enhancement in in vitro digestibility. In vitro dry matter digestibility (IVDMD) of $P$. ostreatus axenic culture and coculture treated rice straw were improved compared to the control except for $P$. chrysosporium, which has an IVDMD lower than the untreated rice straw although after treating rice straw with $P$. chrysosporium, there was a substantial reduction in lignin compared to the untreated straw. The severe loss of DM and cellulose may have weakened its nutritional value resulting in a lowered IVDMD value. Thus, though ADL degradation exposed the holocellulose, there was simultaneous degradation of the exposed cellulose by $P$. chrysosporium, which considerably reduced the amount of cellulose available for rumen microorganism's digestion hence a further decline in substrate digestibility compared to untreated rice straw. Treatment of naked oat with $P$. chrysosporium led to a further decline in IVDMD compared to the untreated straw ${ }^{17}$ which is similar to the result of the present study. Therefore, even though $P$. chrysosporium has excellent ADL degradation ability compared to untreated straw, it cannot improve the biomass's nutritional value after fermentation, indicating that it is not suitable for pretreatment of rice straw.

$P$. ostreatus is an edible mushroom that selectively degrades lignin than cellulose in lignocellulosic biomass via a mechanism that differs from other fungi. In this study, the ADL degradation by $P$. ostreatus was markedly the highest, but cellulose levels decreased slightly after pretreatment. Correspondingly, a considerable improvement was observed in the IVDMD of straw pretreated with $P$. ostreatus. This study's result is similar to the improvement in dry matter digestibility previously reported by Atalar and Çetinkaya ${ }^{29}$. This is because a reduction in lignin wasn't accompanied by excessive cellulose loss; as such, rumen microorganism had access to much cellulose for digestion. IVDMD of coculture treated rice straw improved markedly than $P$. chrysosporium axenic culture and untreated straw. This observation is similar to the increased in vitro digestibility achieved on spruce wood degradation via a $P$. chrysosporium and $P$. ostreatus coculture ${ }^{30}$. The reason is that the coculture could act synergistically in ensuring a much higher degradation of lignin with moderate loss of cellulose.

Volatile fatty acid (VFAs) is the last carbohydrate fermentation product and is the ruminants' energy reserves. As the prime originator of energy supply for ruminants, VFA concentration directly reflects the digestibility of feed. The different amounts of total VFA in various fungal treatment groups aligned with the observed IVDMD and gas production. In the present study, SSF of rice straw treated with $P$. ostreatus 
increased the total VFA whiles $P$. chrysosporium resulted in a decline which is coherent with the findings of Niu, et al. ${ }^{19}$. The reason for this observation is that substrate with higher digestibility value implies more access to fermentable carbohydrates by rumen microbes, which in turns yields higher total VFA compared with substrate with lower digestibility value. The coculture had the ability to increase the total VFA of rice straw although it did not surpass $P$. ostreatus treated rice straw. This might be due to synergistic enzyme action which ensured an efficient hydrolysis of cell wall constituent.

The value of a feed is denoted by its total VFA yields along with its molar proportion, particularly the A:P fraction, which is indicative of the balance between roughages and concentrates. In the rumen, a rise in feed efficiency is linked with a lower A:P ratio as acetate within animal tissue is non gluconeogenic and copious amount is oxidized or utilized for lipogenesis ${ }^{31}$. In the present study, P. ostreatus treated rice straw achieved a deceased A:P ratio (2.63), which contradicted the high value (3.27) reported in the study of Zuo, et al. ${ }^{32}$. This variation could originate from the different substrate, incubation period and temperature utilized. P. chrysosporium treated rice straw A:P (3.18) was the highest (worse), and similar to the A:P value (3.55) reported by Niu, et al. ${ }^{19}$. The coculture achieved a decreased A:P ratio compared to $P$. chrysosporium which is an indication of a better feed efficiency via synergistic action compared to the latter. In addition, the lower acetate: propionate is an indicator of an improved ruminal fermentation efficiency as energy is available for rumen microbe's activities. Conversely, higher acetate: propionate of control and $P$. chrysosporium treated rice straw is an indication of energy inefficiency during fermentation.

In vitro gas production is as a result of gas from direct fermentation and indirect gas produced from the buffering of short chain fatty acids (SCFA). Gas production is a function of carbohydrates fermentation to acetate, propionate and butyrate. The volume of gas produced from rumen microbial fermentation of feedstuffs in vitro is closely related to its digestibility ${ }^{33}$. The greater the volume of gas produced the higher the digestibility. From the present result, gas volume increased progressively in all the substrates, but was higher in rice straw treated with $P$. ostreatus. This observation suggests an improvement in the digestibility of the fungi-treated substrate which can be ascribed to the decrease in the fiber components as a result of selective degradation of lignin over cellulose. This observation agrees with the increase in the total volume of gas production (IVGP) reported by Tuyena, et al. ${ }^{34}$.

Although the total IVGP of the $P$. chrysosporium treated rice straw increased, it was the least of all the treatments. This is similar to the decline in total in vitro volume of gas production from wheat straw treated with $P$. chrysosporium ${ }^{18}$. This is because $P$. chrysosporium extensively consumed cell wall polysaccharides which resulted in a lower IVDMD. The IVGP of coculture being superior to control and $P$. chrysosporium is evident of synergistic enzyme action originating from the axenic culture species' combination.

\section{Conclusion}


The evaluation of the interaction between the axenic ligninolytic fungal species showed that mutual intermingling plus inhibition is the prevalent interaction although, the species can grow together in the same medium. The coculture results suggest that the ligninolytic white-rot fungal consortia led to a synergy between the axenic species, which is evident in its capability to degrade lignin without affecting cellulose to a great extent leading to an improved in vitro rumen fermentation characteristic compared to P. chrysosporium axenic culture.

A comparison among the axenic species and coculture showed that the best fiber degradation, in vitro digestibility and VFA percentages were achieved with $P$. ostreatus axenic culture. A consortium of $P$. chrysosporium and $P$. ostreatus axenic culture can be developed as a lignocellulolytic consortium for converting rice straw into a value added fiber product for ruminant nutrition. An in vivo feeding trail using $P$. chrysosporium and $P$. ostreatus coculture treated rice straw is needed to evaluate its on-farm feeding impact and implication.

\section{Declarations}

\section{Data Availability}

The datasets generated during and/or analysed during the current study are available from the corresponding author on reasonable request.

\section{Funding}

China Agriculture Research System of MOF and MARA.

\section{Author contribution statement}

O.D. conceived the experiment(s), conducted the experiment(s), analyzed the results and wrote the manuscript. Z.G. and L.M. contributed to the manuscript revision. All authors read and approved the manuscript.

\section{Competing interests}

The authors declare no competing interests.

\section{References}

1. Oladosu, Y. et al. Fermentation quality and additives: A case of rice straw silage. Biomed Res. Int. 2016, 14, doi:https://doi.org/10.1155/2016/7985167 (2016).

2. Van Soest, P. Rice straw, the role of silica and treatments to improve quality. Anim. Feed Sci. Technol, 130, 137-171 https://doi.org/10.1016/j.anifeedsci.2006.01.023 (2006).

3. Bari, E. et al. Monitoring the cell wall characteristics of degraded beech wood by white-rot fungi: Anatomical, chemical, and photochemical study. Maderas: Cienc. Tecnol, 20, 35-56 
https://doi.org/10.4067/S0718-221X2018005001401 (2018).

4. Have, R. \& Teunissen, P. J. Oxidative mechanisms involved in lignin degradation by white-rot fungi. Chem. Rev, 101, 3397-3414 https://doi.org/10.1021/cr000115I (2001).

5. Janusz, G. et al. Lignin degradation: microorganisms, enzymes involved, genomes analysis and evolution. FEMS Microbiol. Rev, 41, 941-962 https://doi.org/10.1093/femsre/fux049 (2017).

6. Chi, Y., Hatakka, A. \& Maijala, P. Can co-culturing of two white-rot fungi increase lignin degradation and the production of lignin-degrading enzymes? Int. Biodeterior. Biodegradation, 59, 32-39 https://doi.org/10.1016/j.ibiod.2006.06.025 (2007).

7. Chen, K., Tang, J., Xu, B., Lan, S. \& Cao, Y. Degradation enhancement of rice straw by co-culture of Phanerochaete chrysosporium and Trichoderma viride. Sci. Rep, 9, 1-7 https://doi.org/10.1038/s41598-019-56123-5 (2019).

8. Yang, L. et al. Enhanced Lignin Degradation in Tobacco Stalk Composting with Inoculation of WhiteRot Fungi Trametes hirsuta and Pleurotus ostreatus. Waste Biomass Valorization, 11, 3525-3535 https://doi.org/10.1007/s12649-019-00692-z (2020).

9. Kaur, P., Kocher, G. S. \& Taggar, M. S. Development of fungal consortium for the pretreatment of rice straw under optimized solid state and shake flask conditions. Environ. Prog. Sustain. Energy, 38, 635-646 (2019).

10. Rayner, A. D. M. \& Boddy, L. in Advances in Microbial Ecology Vol. 10 (ed. Marshall, K. C.)115166(Springer, 1988).

11. Van Soest, P., Robertson, J. \& Lewis, B. Methods for dietary fiber, neutral detergent fiber, and nonstarch polysaccharides in relation to animal nutrition. J. Dairy Sci, 74, 3583-3597 https://doi.org/10.3168/jds.S0022-0302(91)78551-2 (1991).

12. Menke, K. et al. The estimation of the digestibility and metabolizable energy content of ruminant feedingstuffs from the gas production when they are incubated with rumen liquor in vitro. J. Agric. Sci, 93, 217-222 https://doi.org/10.1017/S0021859600086305 (1979).

13. Windram, O., Stoker, C. \& Denby, K. in Botrytis-the Fungus, the Pathogen and its Management in Agricultural Systems (eds. Fillinger, S. \& Elad, Y.) 335-360(Springer, 2016).

14. Boddy, L. Interspecific combative interactions between wood-decaying basidiomycetes. FEMS Microbiol. Ecol, 31, 185-194 https://doi.org/10.1111/j.1574-6941.2000.tb00683.x (2000).

15. Hammel, K. \& Cullen, D. Role of fungal peroxidases in biological ligninolysis. Curr. Opin. Plant Biol, 11, 349-355 https://doi.org/10.1016/j.pbi.2008.02.003 (2008).

16. Tirado-González, D. N. et al. Production of cellulases and xylanases by white-rot fungi cultured in corn stover media for ruminant feed applications. Anim. Feed Sci. Technol, 221, 147-156 https://doi.org/10.1016/j.anifeedsci.2016.09.001 (2016).

17. Zheng, M. et al. Effect of Four Species of White Rot Fungi on the Chemical Composition and In Vitro Rumen Degradability of Naked Oat Straw. Waste Biomass Valorization, 12, 435-443 https://doi.org/10.1007/s12649-020-00991-w (2021). 
18. Kerem, Z., Friesem, D. \& Hadar, Y. Lignocellulose degradation during solid-state fermentation: Pleurotus ostreatus versus Phanerochaete chrysosporium. Appl. Environ. Microbiol, 58, 1121-1127 https://doi.org/10.1128/AEM.58.4.1121-1127.1992 (1992).

19. Niu, D. et al. Treatment using white rot fungi changed the chemical composition of wheat straw and enhanced digestion by rumen microbiota in vitro. Anim. Feed Sci. Technol, 237, 46-54 https://doi.org/10.1016/j.anifeedsci.2018.01.005 (2018).

20. Tuyen, V., Cone, J., Baars, J., Sonnenberg, A. \& Hendriks, W. Fungal strain and incubation period affect chemical composition and nutrient availability of wheat straw for rumen fermentation. Bioresour. Technol, 111, 336-342 https://doi.org/10.1016/j.biortech.2012.02.001 (2012).

21. Salvachúa, D., Prieto, A., Vaquero, M. E., Martínez, Ã. T. \& Martínez, M. J. Sugar recoveries from wheat straw following treatments with the fungus Irpex lacteus. Bioresour. Technol, 131, 218-225 https://doi.org/10.1016/j.biortech.2012.11.089 (2013).

22. Yadav, A. N., Singh, S., Mishra, S. \& Gupta, A. Recent Advancement in White Biotechnology Through Fungi: Perspective for Value-Added Products and Environments (Springer, 2019).

23. Nie, H. et al. Comparison of in vitro digestibility and chemical composition among four crop straws treated by Pleurotus ostreatus. Asian-Australas. J. Anim. Sci, 33, 24 https://doi.org/10.5713/ajas.18.0023 (2018).

24. Cohen, R., Persky, L. \& Hadar, Y. Biotechnological applications and potential of wood-degrading mushrooms of the genus Pleurotus. Appl. Environ. Microbiol, 58, 582-594 https://doi.org/10.1007/s00253-002-0930-y (2002).

25. Bari, E. et al. Characterizing Fungal Decay of Beech Wood: Potential for Biotechnological Applications. Microorganisms, 9, 247 https://doi.org/10.3390/microorganisms9020247 (2021).

26. Kato, S., Haruta, S., Cui, Z. J., Ishii, M. \& Igarashi, Y. Stable coexistence of five bacterial strains as a cellulose-degrading community. Appl. Environ. Microbiol, 71, 7099-7106 https://doi.org/10.1128/AEM.71.11.7099-7106.2005 (2005).

27. Khonkhaeng, B. \& Cherdthong, A. Improving Nutritive Value of Purple Field Corn Residue and Rice Straw by Culturing with White-Rot Fungi. J. Fungi, 6, 69 https://doi.org/10.3390/jof6020069 (2020).

28. Valente, T. N. P. et al. Ruminal microorganism consideration and protein used in the metabolism of the ruminants: A review. Afr. J. Microbiol. Res, 10, 456-464 https://doi.org/10.5897/AJMR2016.7627 (2016).

29. Atalar, A. \& Çetinkaya, N. Treatment of Corn Straw with Pleurotus ostreatus, Pleurotus eryngii and Lentinula edodes to Improve the Digestibility of the Lignocellulosic Complex. J. anatol. environ. animal sci, 5, 765-771 https://doi.org/10.35229/jaes.812010 (2020).

30. Asiegbu, F., Paterson, A. \& Smith, J. The effects of co-fungal cultures and supplementation with carbohydrate adjuncts on lignin biodegradation and substrate digestibility. World J. Microbiol. Biotechnol, 12, 273-279 https://doi.org/10.1007/BF00360927 (1996).

31. Wu, H., Meng, Q. \& Yu, Z. Evaluation of ferric oxide and ferric citrate for their effects on fermentation, production of sulfide and methane, and abundance of select microbial populations using in vitro 
rumen cultures. Bioresour. Technol, 211, 603-609 https://doi.org/10.1016/j.biortech.2016.03.126 (2016).

32. Zuo, S. et al. Effect of Irpex lacteus, Pleurotus ostreatus and Pleurotus cystidiosus pretreatment of corn stover on its improvement of the in vitro rumen fermentation. J. Sci. Food Agric. 98, 4287-4295, doi:https://doi.org/10.1002/jsfa.8951 (2018).

33. Cone, J. W. \& van Gelder, A. H. Influence of protein fermentation on gas production profiles. Anim. Feed Sci. Technol, 76, 251-264 https://doi.org/10.1016/S0377-8401(98)00222-3 (1999).

34. Tuyena, V. et al. Effect of fungal treatments of fibrous agricultural by-products on chemical composition and in vitro rumen fermentation and methane production. Bioresour. Technol, 129, 256263 https://doi.org/10.1016/j.biortech.2012.10.128 (2013).

\section{Figures}

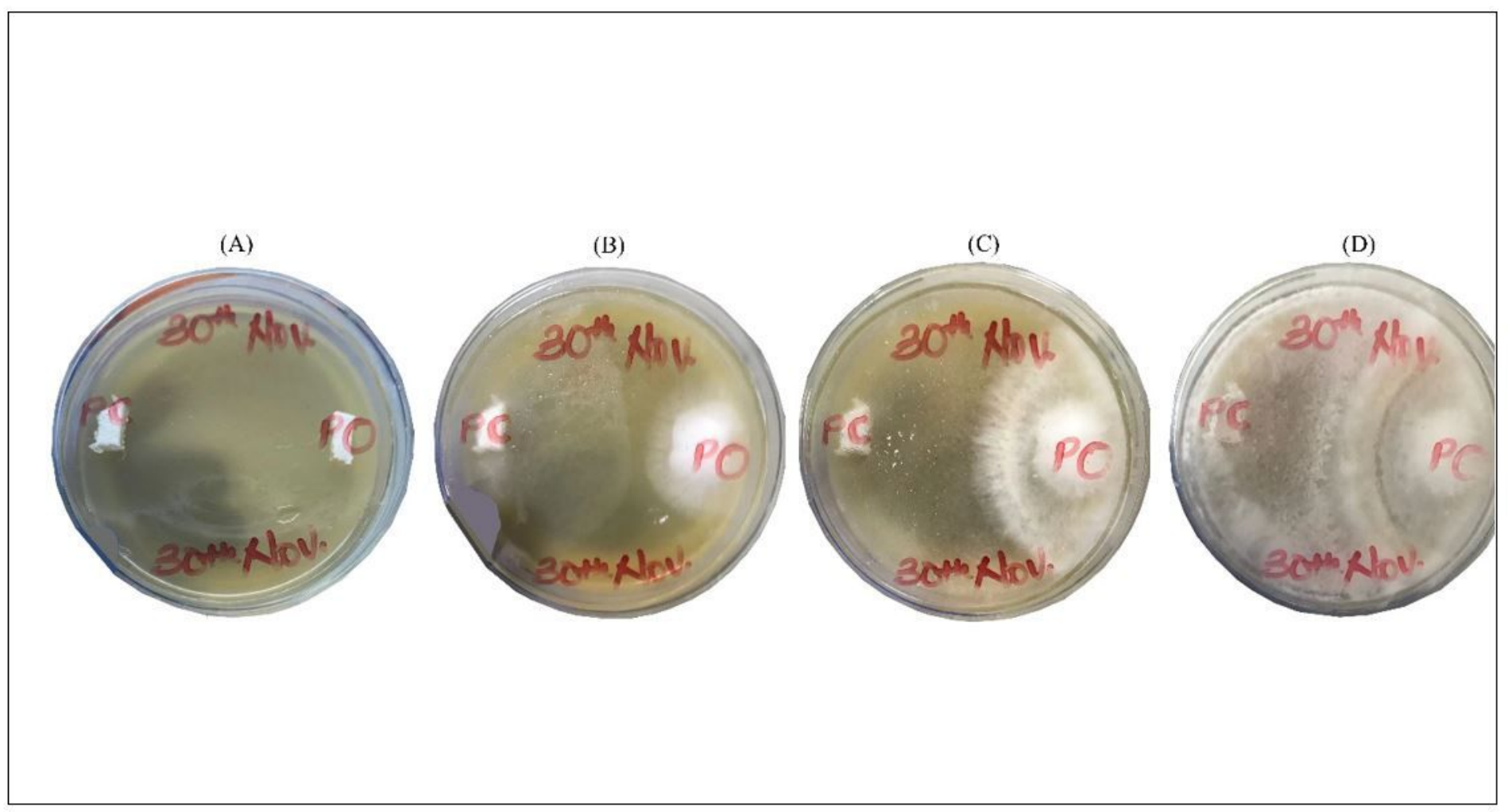

\section{Figure 1}

Mycelia interaction after 7 days of co-incubation between P. chrysosporium and P. ostreatus fungal isolates malt extract solid plate medium: (A) 1 day (B) 3 days (C) 5 days (D) 7 days. 


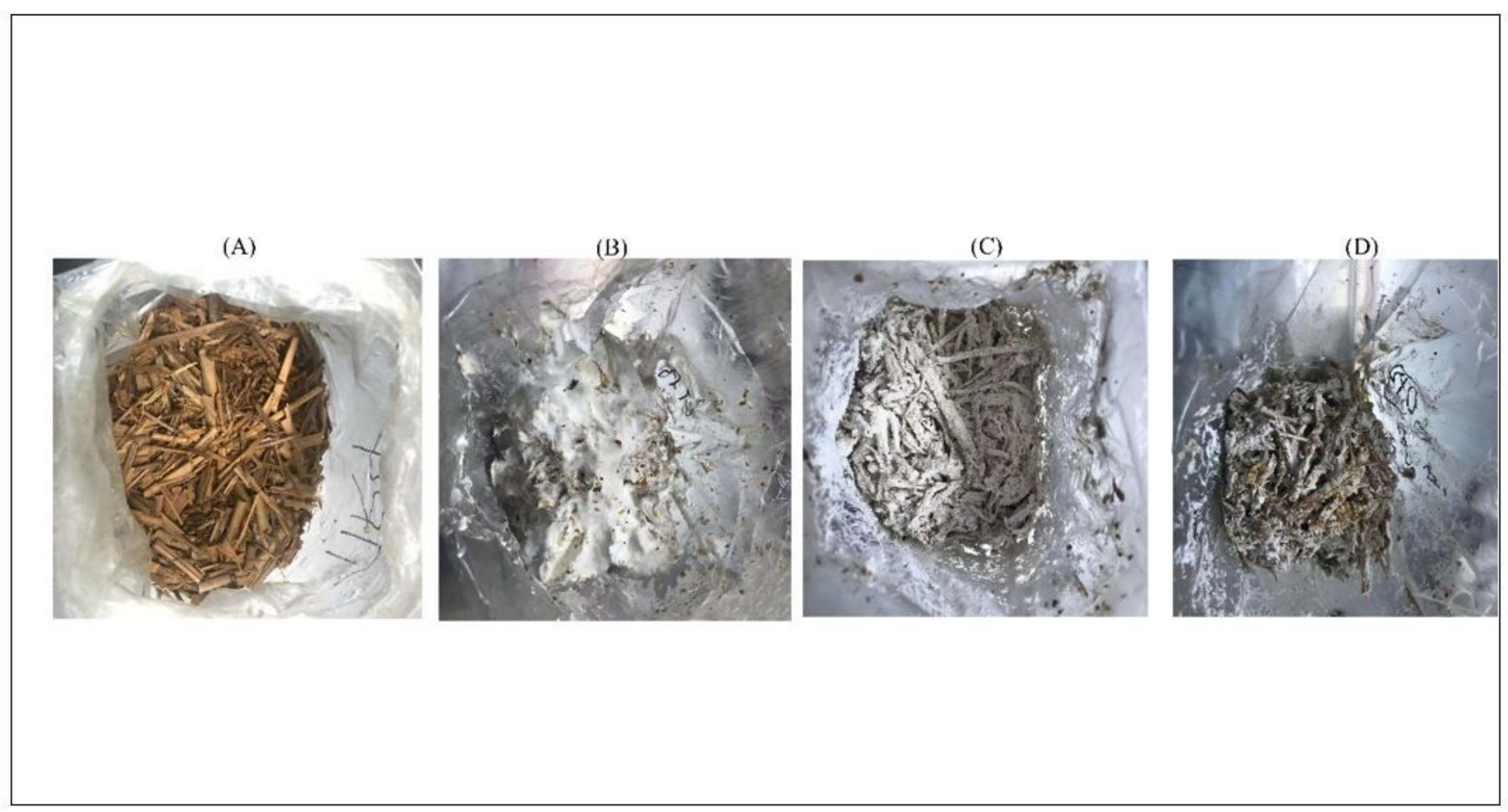

Figure 2

Rice straw after 30 days incubation: (A) control (B) P. ostreatus (C) P. chrysosporium (D) coculture. 